\title{
Controlling the Phase Transition in Superfluid Helium-3
}

\author{
Researchers demonstrate that they can suppress the formation of defects \\ that appear in superfluid helium-3 when it undergoes a continuous phase \\ transition, allowing them to influence the form of the system's final phase.
}

By Jacek Dziarmaga

W

hen a system that can be described by the 2D Ising model cools, it transitions from having a paramagnetic phase to having a ferromagnetic one via a continuous phase transition. During such a phase transition, magnetic defects can form in the material, creating a nonuniform final ferromagnetic phase. Juho Rysti of Aalto University, Finland, and colleagues now show that they can suppress the formation of these defects in superfluid helium-3-when it undergoes a 3D continuous phase

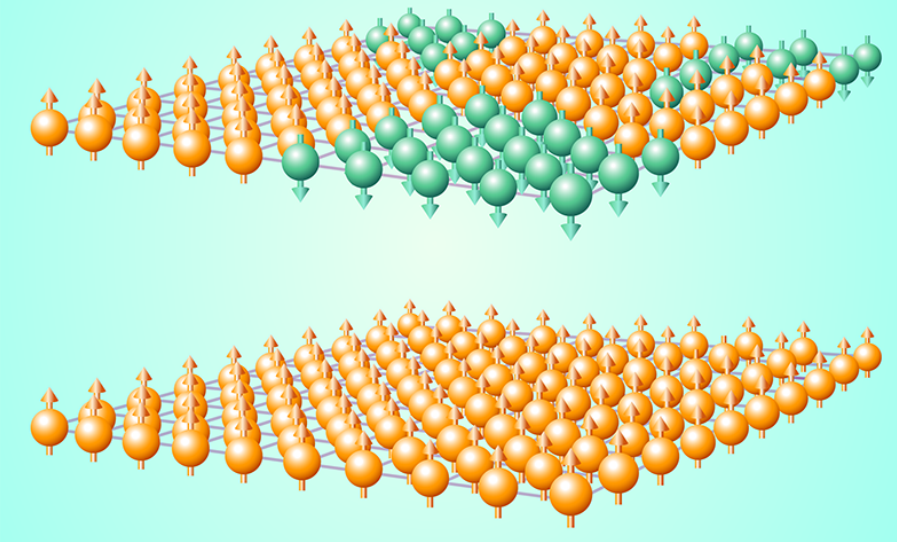

Figure 1: Applying a bias, such as a magnetic field, to a magnetic material as it undergoes a continuous phase transition can force the spins in the material to all align in the same direction (bottom). Without that bias, the spins do not align and instead the material contains a patchwork of oppositely pointing spin domains (top). Credit: APS/Carin Cain transition-by applying a symmetry-breaking bias field to the material [1]. This technique could also be applied to materials undergoing quantum phase transitions, where the appearance of defects can demolish quantum states prepared by adiabatic evolution.

The high-temperature paramagnetic and low-temperature ferromagnetic phases of the 2D Ising model differ by their symmetry: The paramagnetic phase is symmetric - the phase looks the same if the pointing direction of its spins are simultaneously reversed-while the two ferromagnetic phases of the model are symmetry broken. As a 2D Ising system cools from its paramagnetic phase to a ferromagnetic one, it has to choose which of the two ferromagnetic phases it will transition to, and the evolution of the system slows down near the critical point as the system tries to make this choice.

This "critical" slowing down causes different parts of the system to move out of thermal equilibrium with each other, something that allows different parts of the system to make independent choices of their magnetization. If the different parts can communicate with each other, the choices can be coordinated, which is more likely for slower cooling rates. Slower cooling rates thus lead to larger domains of one or other of the ferromagnetic phases, with the size of the domains being quantifiable using the Kibble-Zurek-mechanism theory [2-4]. That said, after the phase transition occurs, the final ferromagnetic phase of the system is almost never uniform but is rather a mosaic of domains of the two ferromagnetic phases (Fig. 1).

The outcome of the phase transition can be made more uniform 
by applying a magnetic field to the system. For example, if this field points upward as the system cools, the decision will be biased toward the ferromagnetic phase that has spins pointing up. The bias is ineffective for very fast cooling rates because there is not enough time for the field to leave its imprint on the phase of the system. So how slow should the cooling rate be for the bias to be effective in ensuring a uniform ferromagnetic phase? The answer comes again from a generalization of the Kibble-Zurek-mechanism theory, which predicts that the maximal cooling rate scales with the bias strength [5]. The new experiment from Rysti and colleagues shows that when the cooling rate is slow enough, the final phase of the system is an equilibrium ferromagnetic one without any domains-the first time that has been seen experimentally.

Rysti and his colleagues study a continuous symmetry-breaking phase transition of superfluid helium-3 [1]. Superfluid helium-3 has more complex magnetic behavior than that of the 2D Ising model: Its "spins" can point in a continuum of directions rather than just up and down, and they can wind into quantized vortices. The nonequilibrium ferromagnetic phase of superfluid helium-3 is a tangle of such vortices, whose density scales with a power of the cooling rate.

In their experiments, the team investigated this scaling behavior by cooling the superfluid using a 3D cryostat and then detecting the orientation of its spins using nuclear magnetic resonance (NMR) coils. In the space between the NMR coils, where the superfluid helium-3 is held, they placed an array of long, thin columns (they call them solid strands), which trap the superfluid's vortices.

The experiment shows that when a bias is applied to the system -the team use both a magnetic field for the bias and also spin-orbit coupling-the power law relating the density of vortices to the cooling rate can break down. Specifically, Rysti and colleagues find that this breakdown happens when the cooling rate falls below a threshold value that is proportional to a power of the bias, with the exponent of the power law being a combination of the universal critical exponents for the transition. Cooling at rates below this threshold value, they find that the density of vortices decays exponentially with cooling time such that the final phase becomes a uniform, equilibrium one.
The team found that the 1-mT bias that they apply is effective only near the phase transition's critical temperature where the system is most susceptible to small perturbations, and even the tiniest of biases can influence the orientation of the spins. They also found that the transition is adiabatic, and as such, they show that cooling with a bias is an efficient way to achieve an adiabatic transition with a finite cooling rate, something that could allow use of the method for adiabatic quantum state preparation in an adiabatic quantum simulator, for example.

The idea of such a simulator is to evolve a system adiabatically from a "simple" ground state to a more interesting one that cannot be calculated analytically or with a classical computer. If successfully prepared in a quantum simulator, the properties of such a state could simply be measured. Unfortunately, these two ground states are often different enough that to move the system from one to the other requires that the system goes through a quantum phase transition. That means that any adiabatic simulator must be able to evolve a system that is close to its quantum critical point.

This evolution can be described by a quantum generalization of the Kibble-Zurek-mechanism theory, which predicts that, because of a closing of the energy gap of the system at the quantum critical point, excitation of the system is inevitable [6, 7]. It is predicted, however, that in symmetry-breaking transitions these excitations can be suppressed by applying a bias while the system is crossing the quantum critical point [5]. The bias is too weak to affect the properties of the final ground state but is large enough to prevent excitations that would destroy the ground state. The new demonstration by Rysti and colleagues shows that this should be experimentally possible, opening the door to many future experiments on this topic.

Jacek Dziarmaga: Institute of Theoretical Physics, Jagiellonian University, Krakow, Poland

\section{REFERENCES}

1. J. Rysti et al., "Suppressing the Kibble-Zurek mechanism by a symmetry-violating bias," Phys. Rev. Lett. 127, 115702 (2021).

2. T. W. B. Kibble, "Topology of cosmic domains and strings," J. Phys. A 9, 1387 (1976); T. W. B. Kibble, "Some implications of a cosmological phase transition,” Phys. Rep. 67, 183 (1980); T. W. B. Kibble, "Phase-transition dynamics in the lab and the universe," Phys. Today 60, 47 (2007). 
3. W. H. Zurek, "Cosmological experiments in superfluid helium?" Nature 317, 505 (1985); W. H. Zurek, "Cosmic strings in laboratory superfluids and the topological remnants of other phase transitions," Acta Phys. Polon. B 24, 1301 (1993); W. H. Zurek, "Cosmological experiments in condensed matter systems," Phys. Rep. 276, 177 (1996).

4. A. del Campo and W. H. Zurek, "Universality of phase transition dynamics: Topological defects from symmetry breaking," Int. J. Mod. Phys. A 29, 1430018 (2014).
5. M. M. Rams et al., "Symmetry breaking bias and the dynamics of a quantum phase transition,” Phys. Rev. Lett. 123, 130603 (2019).

6. J. Dziarmaga, "Dynamics of a quantum phase transition and relaxation to a steady state," Adv. Phys. 59, 1063 (2010).

7. A. Polkovnikov et al., "Colloquium: Nonequilibrium dynamics of closed interacting quantum systems," Rev. Mod. Phys. 83, 863 (2011). 